\title{
Existence of solutions of fractional boundary value problems with $p$-Laplacian operator
}

\author{
Nazim I Mahmudov and Sinem Unul ${ }^{*}$
}

\section{"Correspondence:}

sinem.unul@emu.edu.tr Eastern Mediterranean University, Gazimagusa, TRNC, Mersin 10, Turkey

\begin{abstract}
In this paper, the existence of the solutions of the fractional differential equation with $p$-Laplacian operator and integral conditions is discussed. By Green's functions and the fixed point theorems, we state and prove the existence and uniqueness results of the problem. Two examples are given to illustrate the results.
\end{abstract}

Keywords: existence and uniqueness; fractional calculus; $p$-Laplacian

\section{Introduction}

Differential equations are useful in modern physics, engineering, and in various fields of science. In these days, the theory on existence and uniqueness of boundary value problems of linear and/or nonlinear fractional equations has attracted the attention of many authors. There are comprehensive studies in this area. At the same time, it is known that the $p$ Laplacian operator is also used in analyzing mechanics, physics and dynamic systems, and the related fields of mathematical modeling. However, there are few studies of the existence and uniqueness of boundary conditions of fractional differential equations with the $p$-Laplacian operator, see [1-27] and the references therein.

Zhang et al. [4] studied the eigenvalue problem for a class of singular $p$-Laplacian fractional differential equations involving a Riemann-Stieltjes integral boundary condition:

$$
\begin{aligned}
& -D_{t}^{\beta}\left(\phi_{p}\left(D_{t}^{\alpha} x\right)\right)(t)=\lambda f(t, x(t)), \quad t \in(0,1), \\
& x(0)=0, \quad D_{t}^{\alpha} x(0)=0, \\
& x(1)=\int_{0}^{1} x(s) d A(s),
\end{aligned}
$$

where $D_{t}^{\beta}$ and $D_{t}^{\alpha}$ are standard Riemann-Liouville derivatives with $1<\alpha \leq 2,0<\beta \leq 1$, $A$ is a function of the bounded variation, and $\int_{0}^{1} x(s) d A(s)$ is the standard Riemann-Stieltjes integral. In their study, the results are based on upper and lower solution methods and the Schauder fixed point theorem.

In [5], Su et al. studied the existence criteria of non-negative solutions of nonlinear $p$-Laplacian fractional differential equations with first order derivative,

(c) 2015 Mahmudov and Unul. This article is distributed under the terms of the Creative Commons Attribution 4.0 International License (http://creativecommons.org/licenses/by/4.0/), which permits unrestricted use, distribution, and reproduction in any medium, provided you give appropriate credit to the original author(s) and the source, provide a link to the Creative Commons license, and indicate if changes were made. 


$$
\left\{\begin{array}{l}
\varphi_{p}\left({ }^{c} D^{\alpha} u(t)\right)=\varphi_{p}(\lambda) f\left(t, u(t), u^{\prime}(t)\right), \quad \text { for } t \in(0,1), \\
k_{0} u(0)-k_{1} u(1)=0, \\
m_{0} u(0)-m_{1} u(1)=0, \\
x^{(r)}(0)=0, \quad r=2,3, \ldots,[\alpha],
\end{array}\right.
$$

where $\varphi_{p}$ is $p$-Laplacian operator, i.e. $\varphi_{p}(s)=|s|^{p-2} s, p>1$, and $\varphi_{p}^{-1}=\varphi_{q}, \frac{1}{p}+\frac{1}{q}=1,{ }^{c} D^{\alpha}$ is the Caputo derivative and we have the function $f\left(t, u, u^{\prime}\right):[0,1] \times[0, \infty) \times(-\infty,+\infty) \rightarrow$ $[0, \infty)$ which satisfies the Carathéodory type conditions. Moreover, the nonlinear alternative of Leray-Schauder type and Banach fixed point theorems are used.

Han et al. [6] studied nonlinear fractional differential equations with $p$-Laplacian operator and boundary value conditions,

$$
\begin{aligned}
& D_{0_{+}}^{\alpha}\left(\varphi_{p}\left(D_{0+}^{\alpha} u(t)\right)\right)+a(t) f(u)=0, \quad \text { for } 0<t<1, \\
& u(0)=\gamma u(\xi)+\lambda, \\
& \varphi_{p}\left(D_{0+}^{\alpha} u(0)\right)=\left(\varphi_{p}\left(D_{0+}^{\alpha} u(1)\right)\right)^{\prime}=\left(\varphi_{p}\left(D_{0+}^{\alpha} u(0)\right)\right),
\end{aligned}
$$

where $0<\alpha \leq 1,2<\beta \leq 3$, and $D_{0+}^{\alpha}, D_{0+}^{\beta}$ are Caputo fractional derivatives, $\varphi_{p}(s)=|s|^{p-2} s$, $p>1$, and $\varphi_{p}^{-1}=\varphi_{q}, \frac{1}{p}+\frac{1}{q}=1$, and the parameters are $0 \leq \gamma<1,0 \leq \xi \leq 1, \lambda>0$. The continuous functions $a:(0,1) \rightarrow[0, \infty)$ and $f:[0, \infty) \rightarrow[0, \infty)$ are given. The Green's function properties and the Schauder fixed point theorem are used.

In [2], Liu et al. studied the solvability of the Caputo fractional differential equation with boundary value conditions involving the $p$-Laplacian operator. The existence and uniqueness of the problem is found by the Banach fixed point theorem. The problem is given in the following:

$$
\left(\varphi_{p}\left(D_{0+}^{\alpha} x(t)\right)\right)^{\prime}=f(t, x(t)), \quad \text { for } t \in(0,1)
$$

with boundary value conditions

$$
\begin{aligned}
& x(0)=r_{0} x(1), \\
& x^{\prime}(0)=r_{1} x^{\prime}(1), \\
& x^{(j)}(0)=0,
\end{aligned}
$$

where $i=2,3, \ldots,[\alpha]-1$. Here, $\varphi_{p}$ is the $p$-Laplacian operator and $D_{0+}^{\alpha}$ is the Caputo fractional derivative, $1<\alpha \in R$, and the nonlinear function $f \in C([0,1] \times R, R)$ is given.

In [7], Lu et al. studied the existence of nonnegative solutions of a nonlinear fractional boundary value problem with the $p$-Laplacian operator:

$$
\begin{aligned}
& D_{0+}^{\beta}\left(\varphi_{p}\left(D_{0+}^{\alpha} u(t)\right)\right)=f(t, u(t)), \quad \text { for } 0<t<1, \\
& u(0)=u^{\prime}(0)=u^{\prime}(1)=0, \\
& D_{0+}^{\alpha} u(0)=D_{0+}^{\alpha} u(1)=0,
\end{aligned}
$$

where $2<\alpha \leq 3,1<\beta \leq 2$, and $D_{0+}^{\alpha}, D_{0+}^{\beta}$ are the standard Riemann-Liouville fractional derivatives. Green's functions, the Guo-Krasnoselskii theorem, and the Leggett-Williams fixed point theorems are used. 
In [1], Wang and Xiang used upper and lower solutions method to find the existence results of at least one non-negative solution of the $p$-Laplacian fractional boundary value problem, which is given in the following:

$$
\begin{aligned}
& D_{0+}^{\gamma}\left(\phi_{p}\left(D_{0+}^{\alpha} u(t)\right)\right)=f(t, u(t)), \quad \text { for } 0<t<1, \\
& u(0)=0, \quad u^{\prime}(1)=a u(\xi), \\
& D_{0+}^{\alpha} u(0)=0, \quad D_{0+}^{\alpha} u(1)=b D_{0+}^{\alpha} u(\eta),
\end{aligned}
$$

where $1<\alpha, \gamma \leq 2,0 \leq a, b \leq 1,0<\xi, \eta<1$, and also $D_{0_{+}}^{\alpha}, D_{0+}^{\gamma}$ are Riemann-Liouville fractional operators.

In this paper, we focus on the existence of solutions of the fractional differential equation

$$
D_{0+}^{\beta} \phi_{p}\left(D_{0+}^{\alpha} u(t)\right)=f\left(t, u(t), D_{0+}^{\gamma} u(t)\right)
$$

with the $p$-Laplacian operator and integral boundary conditions,

$$
\begin{aligned}
& u(0)+\mu_{1} u(1)=\sigma_{1} \int_{0}^{1} g(s, u(s)) d s, \\
& u^{\prime}(0)+\mu_{2} u^{\prime}(1)=\sigma_{2} \int_{0}^{1} h(s, u(s)) d s, \\
& D_{0+}^{\alpha} u(0)=0, \\
& D_{0+}^{\alpha} u(1)=v D_{0+}^{\alpha} u(\eta),
\end{aligned}
$$

where $D_{0+}^{\alpha}, D_{0+}^{\beta}$ are for the Caputo fractional differential equation with $1<\alpha \leq 2,1<\beta \leq 2$, $v, \mu_{i}, \sigma_{i}(i=1,2)$ are non-negative parameters. $f, g, h$ are continuous functions. By the Green's functions and fixed point theorems, we state and prove the existence and uniqueness results of the solutions. Two examples are given to illustrate the results.

\section{Preliminaries}

The basic definitions are given in the following.

Definition 1 The Riemann-Liouville fractional integral of order $\alpha>0$ for a function $f$ : $(0,+\infty) \rightarrow R$ is defined as

$$
I_{0+}^{\alpha} f(t)=\frac{1}{\Gamma(\alpha)} \int_{0}^{t}(t-s)^{\alpha-1} f(s) d s,
$$

provided that the right hand side of the integral is pointwise defined on $(0,+\infty)$ and $\Gamma$ is the gamma function.

Definition 2 The Caputo derivative of order $\alpha>0$ for a function $f:(0,+\infty) \rightarrow R$ is written as

$$
D_{0+}^{\alpha} f(t)=\frac{1}{\Gamma(n-\alpha)} \int_{0}^{t}(t-s)^{n-\alpha-1} f^{(n)}(s) d s,
$$

where $n=[\alpha]+1,[\alpha]$ is the integral part of $\alpha$. 
Lemma 3 Let $u \in C(0,1) \cap L^{1}(0,1)$ with the fractional derivative of order $\alpha>0$ that belongs to $C(0,1) \cap L^{1}(0,1)$. Then

$$
I_{0+}^{\alpha} D_{0+}^{\alpha} u(t)=u(t)+c_{1} t^{\alpha-1}+c_{2} t^{\alpha-2}+\cdots+c_{n} t^{\alpha-n},
$$

for $c_{i} \in R(i=1,2, \ldots, n)$, where $n$ is the smallest integer greater than or equal to $\alpha$.

Lemma 4 Let $\alpha>0$. Then the differential equation $D_{0+}^{\alpha} f(t)=0$ has solutions

$$
f(t)=k_{0}+k_{1} t+k_{2} t^{2}+\cdots+k_{n-1} t^{n-1}
$$

and

$$
I_{0+}^{\alpha} D_{0+}^{\alpha} f(t)=f(t)+k_{0}+k_{1} t+k_{2} t^{2}+\cdots+k_{n-1} t^{n-1}
$$

where $k_{i} \in R$ and $i=1,2, \ldots, n=[\alpha]+1$.

The Caputo fractional derivative of order $n-1<\alpha<n$ for $t^{\gamma}$ is given by

$$
D_{0+}^{\alpha} t^{\gamma}= \begin{cases}\frac{\Gamma(\gamma+1)}{\Gamma(\gamma-\alpha+1)} t^{\gamma-\alpha}, & \gamma \in N \text { and } \gamma \geq n \text { or } \gamma \notin N \text { and } \gamma>n-1, \\ 0, & \gamma \in\{0,1, \ldots, n-1\} .\end{cases}
$$

Also, for brevity, we set

$$
\begin{aligned}
& \omega_{1}=\frac{\sigma_{1}}{1+\mu_{1}}-\frac{\sigma_{2} \mu_{1}}{\left(1+\mu_{1}\right)\left(1+\mu_{2}\right)}, \quad \omega_{2}=\frac{\sigma_{2}}{1+\mu_{2}}, \\
& c_{1}(\eta)=\frac{v^{p-1} \eta^{\beta-1}}{\left(1-v^{p-1} \eta\right) \Gamma(\beta+1)}, \quad L=c t^{\beta-1} c_{1}(\eta) .
\end{aligned}
$$

We use the following properties of the $p$-Laplacian operator: $\phi_{p}(u)=|u|^{p-2} u, p>1$, and $\phi_{p}^{-1}=\phi_{q}, \frac{1}{p}+\frac{1}{q}=1$.

(L1) If $1<p<2, u v>0,|u|,|v| \geq r>0$, then

$$
\left|\phi_{p}(u)-\phi_{p}(v)\right| \leq(p-1) r^{p-2}|u-v|
$$

(L2) If $p>2,|u|,|v| \leq \mathbb{R}$ then

$$
\left|\phi_{p}(u)-\phi_{p}(v)\right| \leq(p-1) R^{p-2}|u-v|
$$

We define two Green's functions $G(t, s)$ and $H(t, s)$,

$$
G(t, s)=\left\{\begin{array}{cc}
\frac{(t-\tau)^{\alpha-1}}{\Gamma(\alpha)}-\left(\frac{\mu_{1}\left(1+\mu_{2}\right)+t \mu_{2}\left(1+\mu_{1}\right)}{\left(1+\mu_{1}\right)\left(1+\mu_{2}\right)}\right) \frac{(1-\tau)^{\alpha-1}}{\Gamma(\alpha)} & \\
+\frac{\mu_{1} \mu_{2}(1-\tau)^{\alpha-2}}{\left(1+\mu_{1}\right)\left(1+\mu_{2}\right) \Gamma(\alpha-1)}, & t \geq \tau, \\
-\left(\frac{\mu_{1}\left(1+\mu_{2}\right)+t \mu_{2}\left(1+\mu_{1}\right)}{\left(1+\mu_{1}\right)\left(1+\mu_{2}\right)}\right) \frac{(1-\tau)^{\alpha-1}}{\Gamma(\alpha)} & t \leq \tau, \\
+\frac{\mu_{1} \mu_{2}(1-\tau)^{\alpha-2}}{\left(1+\mu_{1}\right)\left(1+\mu_{2}\right) \Gamma(\alpha-1)}, &
\end{array}\right.
$$


and

$$
H(t, s)= \begin{cases}\frac{[(t-s)]^{\beta-1}}{\Gamma(\beta)}-\frac{t(1-s)^{\beta-1}}{\left(1-v^{p-1} \eta\right) \Gamma(\beta)}, & 0 \leq s \leq t \leq 1, \eta \leq s, \\ \frac{[(t-s)]^{\beta-1}}{\Gamma(\beta)}-\frac{t(1-s)^{\beta-1}}{\left(1-v^{p-1} \eta\right) \Gamma(\beta)}+\frac{t v^{p-1}(\eta-s)^{\beta-1}}{\left(1-v^{p-1} \eta\right) \Gamma(\beta)}, & 0 \leq s \leq t \leq 1, \eta \geq s \\ \frac{-t(1-s)^{\beta-1}}{\left(1-v^{p-1} \eta\right) \Gamma(\beta)}, & 0 \leq t \leq s \leq 1, \eta \leq s, \\ \frac{-t(1-s)^{\beta-1}}{\left(1-v^{p-1} \eta\right) \Gamma(\beta)}+\frac{t v^{p-1}(\eta-s)^{\beta-1}}{\left(1-v^{p-1} \eta\right) \Gamma(\beta)}, & 0 \leq t \leq s \leq 1, \eta \geq s .\end{cases}
$$

Lemma 5 Let $f, g, h \in C(0,1)$, and with $1<\alpha \leq 2$ we have the following fractional boundary value problem:

$$
\begin{aligned}
& D_{0+}^{\beta} \phi_{p}\left(D_{0+}^{\alpha} u(t)\right)=f(t), \\
& \left\{\begin{array}{l}
u(0)+\mu_{1} u(1)=\sigma_{1} \int_{0}^{1} g(s) d s, \\
u^{\prime}(0)+\mu_{2} u^{\prime}(1)=\sigma_{2} \int_{0}^{1} h(s) d s,
\end{array}\right. \\
& D_{0+}^{\alpha} u(0)=0, \\
& D_{0+}^{\alpha} u(1)=v D_{0+}^{\alpha} u(\eta),
\end{aligned}
$$

it has a unique solution which is given by

$$
(\mathcal{T} u)(t)=\int_{0}^{t} G(t, s) \phi_{q}\left(\int_{0}^{1} H(t, \tau) f(\tau) d \tau\right) d s+\omega_{1}+\omega_{2} t
$$

with

$$
\omega_{1}=\frac{\sigma_{1}}{1+\mu_{1}}-\frac{\sigma_{2} \mu_{1}}{\left(1+\mu_{1}\right)\left(1+\mu_{2}\right)} \quad \text { and } \quad \omega_{2}=\frac{\sigma_{2}}{1+\mu_{2}} .
$$

Proof By applying $I_{0+}^{\beta}$ to both sides of (4), we get

$$
\begin{aligned}
& \phi_{p}\left(D_{0+}^{\alpha} u(t)\right)=\int_{0}^{t} \frac{(t-s)^{\beta-1}}{\Gamma(\beta)} f(s) d s-b_{1}-b_{2} t, \quad b_{1}, b_{2} \in R, \\
& D_{0+}^{\alpha} u(t)=\phi_{q}\left(\int_{0}^{t} \frac{(t-s)^{\beta-1}}{\Gamma(\beta)} f(s) d s-b_{1}-b_{2} t\right) .
\end{aligned}
$$

Using the boundary conditions $D_{0_{+}}^{\alpha} u(0)=0$ and $D_{0_{+}}^{\alpha} u(1)=v D_{0+}^{\alpha} u(\eta)$, we have

$$
\phi_{q}\left(-b_{1}\right)=0 \quad \Longrightarrow \quad b_{1}=0
$$

and secondly,

$$
\begin{aligned}
\phi_{q}\left(\int_{0}^{1} \frac{(1-s)^{\beta-1}}{\Gamma(\beta)} f(s) d s-b_{2}\right) & =v \phi_{q}\left(\int_{0}^{\eta} \frac{(\eta-s)^{\beta-1}}{\Gamma(\beta)} f(s) d s-b_{2} \eta\right) \\
& =\phi_{q}\left(v^{\frac{1}{q-1}}\left(\int_{0}^{\eta} \frac{(\eta-s)^{\beta-1}}{\Gamma(\beta)} f(s) d s-b_{2} \eta\right)\right) .
\end{aligned}
$$

Moreover, since $\phi_{p}$ is one-to-one,

$$
I_{0+}^{\beta} f(1)-b_{2}=v^{p-1}\left(I_{0+}^{\beta} f(\eta)-b_{2} \eta\right)=v^{p-1} I_{0+}^{\beta} f(\eta)-v^{p-1} b_{2} \eta,
$$




$$
I_{0+}^{\beta} f(1)-v^{p-1} I_{0+}^{\beta} f(\eta)=\left(1-v^{p-1} \eta\right) b_{2} .
$$

Then

$$
\begin{aligned}
b_{2} & =\frac{1}{\left(1-v^{p-1} \eta\right)} I_{0+}^{\beta} f(1)-\frac{v^{p-1}}{\left(1-v^{p-1} \eta\right)} I_{0+}^{\beta} f(\eta) \\
& =\frac{1}{\left(1-v^{p-1} \eta\right)} \int_{0}^{1} \frac{(1-s)^{\beta-1}}{\Gamma(\beta)} f(s) d s-\frac{v^{p-1}}{\left(1-v^{p-1} \eta\right)} \int_{0}^{\eta} \frac{(\eta-s)^{\beta-1}}{\Gamma(\beta)} f(s) d s .
\end{aligned}
$$

Since $\phi_{p}\left(D_{0_{+}}^{\alpha} u(t)\right)=I_{0+}^{\beta} f(t)-b_{1}-b_{2} t$,

$$
\begin{aligned}
& \phi_{p}\left(D_{0+}^{\alpha} u(t)\right)=\int_{0}^{t} \frac{(t-s)^{\beta-1}}{\Gamma(\beta)} f(s, u(s)) d s-\frac{t}{\left(1-v^{p-1} \eta\right)} \int_{0}^{1} \frac{(1-s)^{\beta-1}}{\Gamma(\beta)} f(s, u(s)) d s \\
& +\frac{t v^{p-1}}{\left(1-v^{p-1} \eta\right)} \int_{0}^{\eta} \frac{(\eta-s)^{\beta-1}}{\Gamma(\beta)} f(s, u(s)) d s \\
& =\int_{0}^{1} H(t, s) f(s) d s \text {, } \\
& D_{0+}^{\alpha} u(t)=\phi_{q}\left(\int_{0}^{1} H(t, s) f(s) d s\right), \\
& u(t)=\int_{0}^{t} \frac{(t-\tau)^{\alpha-1}}{\Gamma(\alpha)} \phi_{q}\left(\int_{0}^{1} H(t, s) f(s) d s\right) d \tau-c_{1}-c_{2} t .
\end{aligned}
$$

By the boundary conditions (5), we get

$$
\begin{aligned}
& -c_{1}+\mu_{1}\left(\int_{0}^{1} \frac{(1-\tau)^{\alpha-1}}{\Gamma(\alpha)} \phi_{p}\left(\int_{0}^{1} H(\tau, s) f(s) d s\right) d \tau-c_{1}-c_{2}\right)=\sigma_{1} \int_{0}^{1} g(s) d s, \\
& \mu_{1} \int_{0}^{1} \frac{(1-\tau)^{\alpha-1}}{\Gamma(\alpha)} \phi_{p}\left(\int_{0}^{1} H(\tau, s) f(s) d s\right) d \tau-c_{2} \mu_{1}-\sigma_{1} \int_{0}^{1} g(s) d s=c_{1}\left(1+\mu_{1}\right), \\
& c_{1}=\frac{\mu_{1}}{\left(1+\mu_{1}\right)} \int_{0}^{1} \frac{(1-\tau)^{\alpha-1}}{\Gamma(\alpha)} \phi_{p}\left(\int_{0}^{1} H(\tau, s) f(s) d s\right) d \tau-c_{2} \frac{\mu_{1}}{\left(1+\mu_{1}\right)} \\
& \quad-\frac{\sigma_{1}}{\left(1+\mu_{1}\right)} \int_{0}^{1} g(s) d s \\
& c_{2}=\frac{\mu_{2}}{\left(1+\mu_{2}\right)} \int_{0}^{1} \frac{(1-\tau)^{\alpha-2}}{\Gamma(\alpha-1)} \phi_{p}\left(\int_{0}^{1} H(\tau, s) f(s) d s\right) d \tau-\frac{\sigma_{2}}{\left(1+\mu_{2}\right)} \int_{0}^{1} h(s) d s .
\end{aligned}
$$

Inserting $c_{2}$ into (8), we get the values of $c_{1}$, and inserting $c_{1}$ and $c_{2}$ into (7), we have

$$
\begin{aligned}
u(t)= & \int_{0}^{t} \frac{(t-\tau)^{\alpha-1}}{\Gamma(\alpha)} \phi_{p}\left(\int_{0}^{1} H(t, s) f(s, u(s)) d s\right) d \tau \\
& -\left(\frac{\mu_{1}\left(1+\mu_{2}\right)+t \mu_{2}\left(1+\mu_{1}\right)}{\left(1+\mu_{1}\right)\left(1+\mu_{2}\right)}\right) \int_{0}^{1} \frac{(1-\tau)^{\alpha-1}}{\Gamma(\alpha)} \phi_{p}\left(\int_{0}^{1} H(\tau, s) f(s, u(s)) d s\right) d \tau \\
& +\frac{\mu_{1} \mu_{2}}{\left(1+\mu_{1}\right)\left(1+\mu_{2}\right)} \int_{0}^{1} \frac{(1-\tau)^{\alpha-2}}{\Gamma(\alpha-1)} \phi_{p}\left(\int_{0}^{1} H(\tau, s) f(s, u(s)) d s\right) d \tau \\
& +\frac{\sigma_{1}}{1+\mu_{1}} \int_{0}^{1} g(s, u(s)) d s-\left(\frac{\sigma_{2} \mu_{1}-t \sigma_{2}\left(1+\mu_{1}\right)}{\left(1+\mu_{1}\right)\left(1+\mu_{2}\right)}\right) \int_{0}^{1} h(s, u(s)) d s .
\end{aligned}
$$


Lemma 6 The functions $G(t, s)$ and $H(t, s)$ are continuous on $[0,1] \times[0,1]$ and $H(t, s)$ satisfies the following properties:

(1) $H(t, s) \leq 0$, for $t, s \in[0,1]$,

(2) $H(t, s) \geq H(s, s)$, for $t, s \in[0,1]$,

(3) the Green's function $H(t, s)$ satisfies the following condition:

$$
0 \leq \int_{0}^{1}|H(t, s)| d s \leq \frac{B(\beta, \beta)}{\left(1-v^{p-1} \eta\right) \Gamma(\beta)},
$$

where $B$ is the Beta function.

Proof The proofs of properties (1)-(2) are given in [1]. Thus we will prove property (3) for any $t, s \in[0,1]$. The Green's function $H(t, s)$ is negative. Therefore,

$$
0 \leq \int_{0}^{1}|H(t, s)| d s \leq \int_{0}^{1}|H(s, s)| d s \leq \frac{B(\beta, \beta)}{\left(1-v^{p-1} \eta\right) \Gamma(\beta)} .
$$

\section{Existence and uniqueness results}

In this section, we state and prove existence and uniqueness results of the fractional BVP (1)-(2) by using the Banach fixed point theorem. Our study concerns the space

$$
C_{\gamma}([0,1], R)=\left\{u \in C([0,1], R), D_{0+}^{\gamma} u \in C([0,1], R)\right\},
$$

which is shown in the form

$$
\|u\|_{\gamma}=\|u\|_{c}+\left\|D_{0+}^{\gamma} u\right\|_{c^{\prime}}
$$

where $\|\cdot\|_{c}$ is the sup norm in $C([0,1], R)$.

The following notations will be used throughout this paper:

$$
\begin{aligned}
& \Delta_{1}=\frac{1}{\Gamma(\alpha+1)}\left[1+\frac{\left|\mu_{1}\right|\left|1+\mu_{2}\right|+\left|\mu_{2}\right|\left|1+\mu_{1}\right|}{\left|1+\mu_{1}\right|\left|1+\mu_{2}\right|}\right]+\frac{1}{\Gamma(\alpha)}\left[\frac{\left|\mu_{1}\right|\left|\mu_{2}\right|}{\left|1+\mu_{1}\right|\left|1+\mu_{2}\right|}\right], \\
& \Delta_{2}=\frac{1}{\Gamma(\alpha-\gamma+1)}\left[1+\frac{\left|\mu_{2}\right|}{\Gamma(2-\gamma)\left|1+\mu_{2}\right|}\right], \\
& \Delta_{g}=\frac{\left|\sigma_{1}\right|}{\left|1+\mu_{1}\right|}, \\
& \Delta_{h_{1}}=\frac{\left|\sigma_{2}\right|\left|\mu_{1}+\right| 1+\mu_{1}||}{\left|1+\mu_{1}\right|\left|1+\mu_{2}\right|}, \quad \Delta_{h_{2}}=\frac{\left|\sigma_{2}\right|}{\Gamma(2-\gamma)\left|1+\mu_{2}\right|} .
\end{aligned}
$$

To state and prove our first result, we pose the following conditions:

(A1) The function $f:[0,1] \times R \times R \rightarrow R$ is jointly continuous.

(A2) There exists a function $l_{f} \in L^{\frac{1}{\tau}}\left([0,1], R^{+}\right)$such that

$$
\left|f\left(t, u_{1}, u_{2}\right)-f\left(t, v_{1}, v_{2}\right)\right| \leq l_{f}(t)\left(\left|u_{1}-v_{1}\right|+\left|u_{2}-v_{2}\right|\right)
$$

for all $\left(t, u_{1}, u_{2}\right),\left(t, v_{1}, v_{2}\right) \in[0,1] \times R \times R$. 
(A3) The functions $g$ and $h$ are jointly continuous and there exists $l_{g}, l_{h} \in L^{1}\left([0,1], R^{+}\right)$ such that

$$
|g(t, u)-g(t, v)| \leq l_{g}(t)|u-v|
$$

and

$$
|h(t, u)-h(t, v)| \leq l_{h}(t)|u-v|
$$

for each $(t, u),(t, v) \in[0,1] \times R$.

Next, we define an operator, $\mathcal{T}_{0}$ which is $\mathcal{T}_{0}: C[0,1] \rightarrow C[0,1]$ as follows:

$$
\mathcal{T}_{0} x(t)=\phi_{q}\left(\int_{0}^{1} H(t, s) f\left(s, x(s), D_{0+}^{\gamma} x(s)\right) d s\right) .
$$

Lemma 7 Assume (A1)-(A3) hold and $q>2$. There exists a constant $l_{T_{0}}>0$ such that

$$
\left|\mathcal{T}_{0} u(t)-\mathcal{T}_{0} v(t)\right| \leq l_{\mathcal{T}_{0}}\|u-v\|_{\gamma},
$$

for all $u, v \in B_{r}$. We have

$$
l_{\mathcal{T}_{0}}=(q-1) L_{H}^{q-2}\left\|l_{f}\right\|_{\infty} \int_{0}^{1}|H(s, s)| d s \leq(q-1) L_{H}^{q-2}\left\|l_{f}\right\|_{\infty} \frac{B(\beta, \beta)}{\left(1-v^{p-1} \eta\right) \Gamma(\beta)} .
$$

Proof If $p>2$ and $t>0$ we have the following estimation:

$$
\begin{aligned}
\left|\int_{0}^{1} H(t, s) f\left(s, u(s), D_{0+}^{\gamma} u(s)\right) d s\right| & \leq \int_{0}^{1}|H(t, s)| f\left(s, u(s), D_{0+}^{\gamma} u(s)\right) \mid d s \\
& \leq \int_{0}^{1}|H(t, s)| l_{f}(s)\left(|u(s)|+\left|D_{0+}^{\gamma} u(s)\right|+|f(s, 0,0)|\right) d s \\
& \leq\left(\left\|l_{f}\right\|_{\infty}\|u\|_{\gamma}+M\right) \int_{0}^{1}|H(s, s)| d s \\
& \leq\left(\left\|l_{f}\right\|_{\infty} r+M\right) \int_{0}^{1}|H(s, s)| d s \\
& =L_{H},
\end{aligned}
$$

where $M=\max _{s \in[0,1]}|f(s, 0,0)|$. Now using the property (L2), we get the desired inequality,

$$
\begin{aligned}
& \left|\left(\mathcal{T}_{0} u\right)(t)-\left(\mathcal{T}_{0} v\right)(t)\right| \\
& \quad=\left|\phi_{q}\left(\int_{0}^{1} H(t, s) f\left(s, u(s), D_{0+}^{\gamma} u(s)\right) d s\right)-\phi_{q}\left(\int_{0}^{1} H(t, s) f\left(s, v(s), D_{0+}^{\gamma} v(s)\right) d s\right)\right| \\
& \quad \leq(q-1) L_{H}^{q-2}\left|\int_{0}^{1} H(t, s)\left(f\left(s, u(s), D_{0+}^{\gamma} u(s)\right)-f\left(s, v(s), D_{0+}^{\gamma} v(s)\right)\right) d s\right| \\
& \quad \leq(q-1) L_{H}^{q-2}\left\|l_{f}\right\|_{\infty}\|u-v\|_{\gamma} \int_{0}^{1}|H(s, s)| d s
\end{aligned}
$$




$$
\begin{aligned}
& \leq(q-1) L_{H}^{q-2}\left\|l_{f}\right\|_{\infty} \frac{B(\beta, \beta)}{\left(1-v^{p-1} \eta\right) \Gamma(\beta)}\|u-v\|_{\gamma} \\
& =l_{\mathcal{T}_{0}}\|u-v\|_{\gamma} .
\end{aligned}
$$

Theorem 8 Assume (A1)-(A3) hold. If

$$
\left\{l_{\mathcal{T}_{0}}\left(\sum_{i=1}^{2} \Delta_{i}\right)+\Delta_{g}\left\|l_{g}\right\|_{1}+\left(\sum_{i=1}^{2} \Delta h_{i}\right)\left\|l_{h}\right\|_{1}\right\}<1,
$$

then our BVP (1)-(2) has a unique solution on $[0,1]$.

Proof Let us define the operator $\mathcal{T}: C_{\gamma}([0,1], R) \rightarrow C_{\gamma}([0,1], R)$ to transform our BVP (1)(2) into a fixed point problem,

$$
\begin{aligned}
(\mathcal{T} u)(t) & \\
= & \int_{0}^{t} \frac{(t-s)^{\alpha-1}}{\Gamma(\alpha)} \mathcal{T}_{0}\left(f\left(s, u(s), D_{0+}^{\gamma} u(s)\right)\right) d s \\
& -\frac{\mu_{1}}{\left(1+\mu_{1}\right)} \int_{0}^{1} \frac{(1-s)^{\alpha-1}}{\Gamma(\alpha)} \mathcal{T}_{0}\left(f\left(s, u(s), D_{0+}^{\gamma} u(s)\right)\right) d s \\
& +\frac{\mu_{1} \mu_{2}}{\left(1+\mu_{1}\right)\left(1+\mu_{2}\right)} \int_{0}^{1} \frac{(1-s)^{\alpha-2}}{\Gamma(\alpha-1)} \mathcal{T}_{0}\left(f\left(s, u(s), D_{0+}^{\gamma} u(s)\right)\right) d s \\
& -\frac{\mu_{2} t}{\left(1+\mu_{2}\right)} \int_{0}^{1} \frac{(1-s)^{\alpha-1}}{\Gamma(\alpha)} \mathcal{T}_{0}\left(f\left(s, u(s), D_{0+}^{\gamma} u(s)\right)\right) d s \\
& +\frac{\sigma_{1}}{\left(1+\mu_{1}\right)} \int_{0}^{1} g(s, u(s)) d s-\frac{\sigma_{2}\left(\mu_{1}-\left(1+\mu_{1}\right) t\right)}{\left(1+\mu_{2}\right)\left(1+\mu_{1}\right)} \int_{0}^{1} h(s, u(s)) d s .
\end{aligned}
$$

Taking the $\gamma$ th fractional derivative, we get

$$
\begin{aligned}
& D_{0+}^{\gamma}(\mathcal{T} u)(t) \\
& \quad=\int_{0}^{t} \frac{(t-s)^{\alpha-\gamma-1}}{\Gamma(\alpha-\gamma)} \mathcal{T}_{0}\left(f\left(s, u(s), D_{0+}^{\gamma} u(s)\right)\right) d s \\
& \quad-\frac{\mu_{2}}{\left(1+\mu_{2}\right)} \frac{t^{1-\gamma}}{\Gamma(2-\gamma)} \int_{0}^{1} \frac{(1-s)^{\alpha-\gamma-1}}{\Gamma(\alpha-\gamma)} \mathcal{T}_{0}\left(f\left(s, u(s), D_{0+}^{\gamma} u(s)\right)\right) d s \\
& \quad+\frac{\sigma_{2}}{\left(1+\mu_{2}\right)} \frac{t^{1-\gamma}}{\Gamma(2-\gamma)} \int_{0}^{1} h(s, u(s)) d s
\end{aligned}
$$

for $t \in[0,1]$. Since $f, g, h$ are continuous, the expression (10) and (11) are well defined. Clearly, the fixed point of the operator $\mathcal{T}$ is the solution of the problem (1)-(2). To show the existence and uniqueness of the solution, the Banach fixed point theorem is used and then we show $\mathcal{T}$ is contraction. We have

$$
\begin{aligned}
& |(\mathcal{T} u)(t)-(\mathcal{T} v)(t)| \\
& \quad \leq \int_{0}^{t} \frac{(t-s)^{\alpha-1}}{\Gamma(\alpha)} l_{\mathcal{T}_{0}}\|u-v\|_{\gamma} d s \\
& \quad+\frac{\left|\mu_{1}\right|}{\left|1+\mu_{1}\right|} \int_{0}^{1} \frac{(1-s)^{\alpha-1}}{\Gamma(\alpha)} l_{\mathcal{T}_{0}}\|u-v\|_{\gamma} d s
\end{aligned}
$$




$$
\begin{aligned}
& +\frac{\left|\mu_{1}\right|\left|\mu_{2}\right|}{\left|1+\mu_{1}\right|\left|1+\mu_{2}\right|} \int_{0}^{1} \frac{(1-s)^{\alpha-2}}{\Gamma(\alpha-1)} l_{\mathcal{T}_{0}}\|u-v\|_{\gamma} d s \\
& +\frac{\left|\mu_{2}\right|}{\left|1+\mu_{2}\right|} \int_{0}^{1} \frac{(1-s)^{\alpha-1}}{\Gamma(\alpha)} l_{\mathcal{T}_{0}}\|u-v\|_{\gamma} d s \\
& +\frac{\left|\sigma_{1}\right|}{\left|1+\mu_{1}\right|} \int_{0}^{1} l_{g}(s)\left(|u(s)-v(s)|+\left|D_{0+}^{\gamma} u(s)-D_{0+}^{\gamma} v(s)\right|\right) d s \\
& +\frac{\left|\sigma_{2}\right|\left|\mu_{1}+\right| 1+\mu_{1}||}{\left|1+\mu_{2}\right|\left|1+\mu_{1}\right|} \int_{0}^{1} l_{h}(s)\left(|u(s)-v(s)|+\left|D_{0+}^{\gamma} u(s)-D_{0+}^{\gamma} v(s)\right|\right) d s \\
& \leq\left\{l _ { \mathcal { T } _ { 0 } } \left(\int_{0}^{t} \frac{(t-s)^{\alpha-1}}{\Gamma(\alpha)} d s+\frac{\left|\mu_{1}\right|\left|1+\mu_{2}\right|+\left|\mu_{2}\right|\left|1+\mu_{1}\right|}{\left|1+\mu_{1}\right|\left|1+\mu_{2}\right|} \int_{0}^{1} \frac{(1-s)^{\alpha-1}}{\Gamma(\alpha)} d s\right.\right. \\
& \left.+\frac{\left|\mu_{1}\right|\left|\mu_{2}\right|}{\left|1+\mu_{1}\right|\left|1+\mu_{2}\right|} \int_{0}^{1} \frac{(1-s)^{\alpha-2}}{\Gamma(\alpha-1)} d s\right) \\
& \left.+\frac{\left|\sigma_{1}\right|}{\left|1+\mu_{1}\right|} \int_{0}^{1} l_{g}(s) d s+\frac{\left|\sigma_{2}\right|\left|\mu_{1}+\right| 1+\mu_{1}||}{\left|1+\mu_{2}\right|\left|1+\mu_{1}\right|} \int_{0}^{1} l_{h}(s) d s\right\}\|u-v\|_{\gamma} \\
& \leq\left\{l_{\mathcal{T}_{0}}\left(\frac{1}{\Gamma(\alpha+1)}+\frac{\left|\mu_{1}\right|\left|1+\mu_{2}\right|+\left|\mu_{2}\right|\left|1+\mu_{1}\right|}{\Gamma(\alpha+1)\left|1+\mu_{1}\right|\left|1+\mu_{2}\right|}+\frac{\left|\mu_{1}\right|\left|\mu_{2}\right|}{\Gamma(\alpha)\left|1+\mu_{1}\right|\left|1+\mu_{2}\right|}\right)\right. \\
& \left.+\frac{\left|\sigma_{1}\right|}{\left|1+\mu_{1}\right|}\left\|l_{g}\right\|_{1}+\frac{\left|\sigma_{2}\right|\left|\mu_{1}+\right| 1+\mu_{1}||}{\left|1+\mu_{2}\right|\left|1+\mu_{1}\right|}\left\|l_{h}\right\|_{1}\right\}\|u-v\|_{\gamma} .
\end{aligned}
$$

By using the Hölder inequality, we get

$$
\begin{aligned}
&|\mathcal{T} u(t)-\mathcal{T} v(t)| \leq\left\{l_{\mathcal{T}_{0}} \Delta_{1}+\Delta_{g}\left\|l_{g}\right\|_{1}+\Delta_{h_{1}}\left\|l_{h}\right\|_{1}\right\}\|u-v\|_{\gamma}, \\
&\left|D_{0+}^{\gamma}(\mathcal{T} u)(t)-D_{0+}^{\gamma}(\mathcal{T} v)(t)\right| \\
& \leq \int_{0}^{t} \frac{(t-s)^{\alpha-\gamma-1}}{\Gamma(\alpha-\gamma)} l_{\mathcal{T}_{0}}\|u-v\|_{\gamma} d s \\
&+\frac{\left|\mu_{2}\right|}{\left|1+\mu_{2}\right|} \frac{t^{1-\gamma}}{\Gamma(2-\gamma)} \int_{0}^{1} \frac{(1-s)^{\alpha-\gamma-1}}{\Gamma(\alpha-\gamma)} l_{\mathcal{T}_{0}}\|u-v\|_{\gamma} d s \\
&+\frac{\left|\sigma_{2}\right|}{\left|1+\mu_{2}\right|} \frac{t^{1-\gamma}}{\Gamma(2-\gamma)} \int_{0}^{1} l_{h}(s)\left(|u(s)-v(s)|+\left|D_{0+}^{\gamma} u(s)-D_{0+}^{\gamma} v(s)\right|\right) d s \\
& \leq\left\{\frac{l_{\mathcal{T}_{0}}}{\Gamma(\alpha-\gamma)} \int_{0}^{t}(t-s)^{\alpha-\gamma-1} d s\right. \\
&+\frac{l_{\mathcal{T}_{0}} t^{1-\gamma}\left|\mu_{2}\right|}{\Gamma(\alpha-\gamma) \Gamma(2-\gamma)\left|1+\mu_{2}\right|} \int_{0}^{1}(1-s)^{\alpha-\gamma-1} d s \\
&\left.+\frac{\left|\sigma_{2}\right| t^{1-\gamma}}{\left|1+\mu_{2}\right| \Gamma(2-\gamma)} \int_{0}^{1} l_{h}(s) d s\right\}\|u-v\|_{\gamma} \\
& \leq\left\{l_{\mathcal{T}_{0}}\left(\frac{1}{\Gamma(\alpha-\gamma+1)}+\frac{\left|\mu_{2}\right|}{\Gamma(\alpha-\gamma+1) \Gamma(2-\gamma)\left|1+\mu_{2}\right|}\right)\right. \\
&\left.+\frac{\left|\sigma_{2}\right|}{\left|1+\mu_{2}\right| \Gamma(2-\gamma)} \int_{0}^{1} l_{h}(s) d s\right\}\|u-v\|_{\gamma} \\
& \leq\left\{\frac{l_{\mathcal{T}_{0}}}{\Gamma(\alpha-\gamma+1)}\left(1+\frac{\left|\sigma_{2}\right|}{\Gamma(2-\gamma)\left|1+\mu_{2}\right|}\right)\right. \\
&\left.+\frac{\left|\mu_{2}\right|}{\left|1+\mu_{2}\right| \Gamma(2-\gamma)}\left\|l_{h}\right\|_{1}\right\}\|u-v\|_{\gamma} .
\end{aligned}
$$


Similarly,

$$
\left|D_{0+}^{\gamma}(\mathcal{T} u(t))-D_{0+}^{\gamma}(\mathcal{T} v(t))\right| \leq\left\{l_{\mathcal{T}_{0}} \Delta_{2}+\Delta_{h_{2}}\left\|l_{h}\right\|_{1}\right\}\|u-v\|_{\gamma}
$$

With the help of (13)-(15), we find that

$$
\begin{aligned}
\| T u & -T v \|_{\gamma} \\
& \leq\left\{l_{\mathcal{T}_{0}}\left(\Delta_{1}+\Delta_{2}\right)+\Delta_{g}\left\|l_{g}\right\|_{1}+\left(\Delta_{h_{1}}+\Delta_{h_{2}}\right)\left\|l_{h}\right\|_{1}\right\}\|u-v\|_{\gamma} \\
& =\left\{l_{\mathcal{T}_{0}}\left(\sum_{i=1}^{2} \Delta_{i}\right)+\Delta_{g}\left\|l_{g}\right\|_{1}+\left(\sum_{i=1}^{2} \Delta_{h_{i}}\right)\left\|l_{h}\right\|_{1}\right\}\|u-v\|_{\gamma} .
\end{aligned}
$$

Thus $\mathcal{T}$ is a contraction mapping by condition (9). By the Banach fixed point theorem, $\mathcal{T}$ has a fixed point which is the solution of the BVP.

\section{Existence results}

Theorem 9 Assume:

(iv) There exist non-decreasing functions $\varphi:[0, \infty) \times[0, \infty) \rightarrow[0, \infty)$ and $\psi_{i}:[0, \infty) \rightarrow$ $[0, \infty), i=1,2$, and the functions $l_{f} \in L^{\frac{1}{\tau}}\left([0,1], R^{+}\right)$and $l_{g}, l_{h} \in L^{1}\left([0,1], R^{+}\right)$such that

$$
\begin{aligned}
& |f(t, u, v)| \leq l_{f}(t) \varphi(|u|+|v|), \\
& |g(t, u)| \leq l_{g}(t) \psi_{1}(|u|), \\
& |h(t, u)| \leq l_{h}(t) \psi_{2}(|u|),
\end{aligned}
$$

for all $t \in[0,1]$ and $u, v \in R$.

(v) There exists a constant $\mathcal{N}>0$ such that

$$
\left[\frac{\mathcal{N}}{\varphi\left(\|u\|_{\gamma}\right) l_{\mathcal{T}_{0}} \sum_{i=1}^{2} \Delta_{i}+\psi_{1}\left(\|u\|_{\gamma}\right)\left\|l_{g}\right\|_{1} \Delta_{g}+\psi_{2}\left(\|u\|_{\gamma}\right)\left\|l_{h}\right\|_{1} \sum_{i=1}^{2} \Delta_{h_{i}}}\right]>1 .
$$

Thus problem (1)-(2) has at least one solution on $[0,1]$.

Proof Let $B_{r}=\left\{u \in C_{\gamma}([0,1], R):\|u\|_{\gamma} \leq r\right\}$.

Step 1: Let the operator $\mathcal{T}: C_{\gamma}([0,1], R) \rightarrow C_{\gamma}([0,1], R)$ be given in (10) which defines $B_{r}$ to be a bounded set. For all $u \in B_{r}$, we get

$$
\begin{aligned}
|(\mathcal{T} u)(t)| & \\
\leq & \frac{\varphi(r)}{\Gamma(\alpha)} l_{\mathcal{T}_{0}} \int_{0}^{t}(t-s)^{\alpha-1} d s \\
& +\frac{\left|\mu_{1}\right|\left|1+\mu_{2}\right|+\left|\mu_{2}\right|\left|1+\mu_{1}\right|}{\left|1+\mu_{1}\right|\left|1+\mu_{2}\right|} \frac{\varphi(r)}{\Gamma(\alpha)} l_{\mathcal{T}_{0}} \int_{0}^{1}(1-s)^{\alpha-1} d s \\
& +\frac{\left|\mu_{1}\right|\left|\mu_{2}\right|}{\left|1+\mu_{1}\right|\left|1+\mu_{2}\right|} \frac{\varphi(r)}{\Gamma(\alpha-1)} l_{\mathcal{T}_{0}} \int_{0}^{1}(1-s)^{\alpha-2} d s \\
& +\frac{\left|\sigma_{1}\right|}{\left|1+\mu_{1}\right|} \psi_{1}(r) \int_{0}^{1}\left|l_{g}(s)\right| d s+\frac{\left|\sigma_{2}\right|\left|\mu_{1}+\right| 1+\mu_{1}||}{\left|1+\mu_{2}\right|\left|1+\mu_{1}\right|} \psi_{2}(r) \int_{0}^{1}\left|l_{h}(s)\right| d s
\end{aligned}
$$


and

$$
\begin{aligned}
& \left|D_{0+}^{\gamma}(\mathcal{T} u)(t)\right| \\
& \leq \leq \frac{\varphi(r)}{\Gamma(\alpha-\gamma)} l_{\mathcal{T}_{0}} \int_{0}^{t}(t-s)^{\alpha-\gamma-1} d s \\
& \quad+\frac{\left|\mu_{2}\right|}{\left|1+\mu_{2}\right|} \frac{t^{1-\gamma}}{\Gamma(2-\gamma)} \frac{\varphi(r)}{\Gamma(\alpha-\gamma)} l_{\mathcal{T}_{0}} \int_{0}^{1}(1-s)^{\alpha-\gamma-1} d s \\
& \quad+\frac{\left|\sigma_{2}\right|}{\left|1+\mu_{2}\right|} \frac{t^{1-\gamma}}{\Gamma(2-\gamma)} \psi_{2}(r) \int_{0}^{1}\left|l_{h}(s)\right| d s .
\end{aligned}
$$

By the Hölder inequality,

$$
\begin{aligned}
& |(\mathcal{T} u)(t)| \\
& \quad \leq \frac{\varphi(r) l_{\mathcal{T}_{0}}}{\Gamma(\alpha+1)}+\frac{\left(\left|\mu_{1}\right|\left|1+\mu_{2}\right|+\left|\mu_{2}\right|\left|1+\mu_{1}\right|\right) \varphi(r) l_{\mathcal{T}_{0}}}{\left|1+\mu_{1}\right|\left|1+\mu_{2}\right| \Gamma(\alpha+1)}+\frac{\left|\mu_{1}\right|\left|\mu_{2}\right| \varphi(r) l_{\mathcal{T}_{0}}}{\left|1+\mu_{1}\right|\left|1+\mu_{2}\right| \Gamma(\alpha)} \\
& \quad+\frac{\left|\sigma_{1}\right| \psi_{1}(r)\left\|l_{g}\right\|_{1}}{\left|1+\mu_{1}\right|}+\frac{\left|\sigma_{2}\right|\left|\mu_{1}+\right| 1+\mu_{1}|| \psi_{2}(r)\left\|l_{h}\right\|_{1}}{\left|1+\mu_{2}\right|\left|1+\mu_{1}\right|} \\
& \quad \leq \varphi(r) l_{\mathcal{T}_{0}} \Delta_{1}+\Delta_{g} \psi_{1}(r)\left\|l_{g}\right\|_{1}+\Delta_{h_{1}} \psi_{2}(r)\left\|l_{h}\right\|_{1}, \\
& \left|D_{0_{+}}^{\gamma}(\mathcal{T} u)(t)\right| \\
& \leq \frac{\varphi(r) l_{\mathcal{T}_{0}}}{\Gamma(\alpha-\gamma+1)}+\frac{\left|\mu_{2}\right| \varphi(r) l_{\mathcal{T}_{0}}}{\left|1+\mu_{2}\right| \Gamma(2-\gamma) \Gamma(\alpha-\gamma+1)}+\frac{\left|\sigma_{2}\right| \psi_{2}(r)\left\|l_{h}\right\|_{1}}{\left|1+\mu_{2}\right| \Gamma(2-\gamma)} \\
& \leq \varphi(r) l_{\mathcal{T}_{0}} \Delta_{2}+\Delta_{h_{2}} \psi_{2}(r)\left\|l_{h}\right\|_{1} .
\end{aligned}
$$

Therefore,

$$
\|(\mathcal{T} u)\|_{\gamma} \leq \varphi(r) l_{\mathcal{T}_{0}}\left(\Delta_{1}+\Delta_{2}\right)+\Delta_{g} \psi_{1}(r)\left\|l_{g}\right\|_{1}+\left(\Delta_{h_{1}}+\Delta_{h_{2}}\right) \psi_{2}(r)\left\|l_{h}\right\|_{1}
$$

Step 2: The families $\left\{(\mathcal{T} u): u \in B_{r}\right\}$ and $\left\{D_{0+}^{\gamma}(\mathcal{T} u): u \in B_{r}\right\}$ are equicontinuous. For $t_{1}<t_{2}$, we get

$$
\begin{aligned}
& \left|(\mathcal{T} u)\left(t_{2}\right)-(\mathcal{T} u)\left(t_{1}\right)\right| \\
& \leq \frac{\varphi(r) l_{\mathcal{T}_{0}}}{\Gamma(\alpha)}\left[\int_{0}^{t_{1}}\left(\left(t_{1}-s\right)^{\alpha-1}+\left(t_{2}-s\right)^{\alpha-1}\right) d s-\int_{t_{1}}^{t_{2}}\left(t_{2}-s\right)^{\alpha-1} d s\right] \\
& \quad+\frac{\left|\mu_{2}\right|\left|t_{2}-t_{1}\right|}{\left|1+\mu_{2}\right|} \frac{\varphi(r) l_{\mathcal{T}_{0}}}{\Gamma(\alpha)} \int_{0}^{t_{1}}(1-s)^{\alpha-1} d s \\
& +\frac{\left|\sigma_{2}\right|\left|1+\mu_{1}\right|\left|t_{2}-t_{1}\right| \psi_{2}(r)}{\left|\mu_{2}\right|\left|1+\mu_{1}\right|} \int_{0}^{1}\left|l_{h}(s)\right| d s \rightarrow 0 \text { as } t_{2} \rightarrow t_{1} .
\end{aligned}
$$

Similarly,

$$
\begin{aligned}
& \left|D_{0+}^{\gamma}(\mathcal{T} u)\left(t_{2}\right)-D_{0+}^{\gamma}(\mathcal{T} u)\left(t_{1}\right)\right| \\
& \quad \leq \frac{\varphi(r) l_{\mathcal{T}_{0}}}{\Gamma(\alpha-\gamma)}\left[\int_{0}^{t_{1}}\left(\left(t_{1}-s\right)^{\alpha-\gamma-1}+\left(t_{2}-s\right)^{\alpha-\gamma-1}\right) d s-\int_{t_{1}}^{t_{2}}\left(t_{2}-s\right)^{\alpha-\gamma-1} d s\right]
\end{aligned}
$$




$$
\begin{aligned}
& +\frac{\varphi(r) l_{\mathcal{T}_{0}}\left|\mu_{2}\right|\left|t_{2}^{1-\gamma}-t_{1}^{1-\gamma}\right|}{\Gamma(\alpha-\gamma)\left|1+\mu_{2}\right| \Gamma(2-\gamma)} \int_{0}^{1}(1-s)^{\alpha-\gamma-1} d s \\
& +\frac{\left|\sigma_{2}\right|\left|t_{2}^{1-\gamma}-t_{1}^{1-\gamma}\right| \psi_{2}(r)}{\left|1+\mu_{2}\right| \Gamma(2-\gamma)} \int_{0}^{1}\left|l_{h}(s)\right| d s \rightarrow 0 \quad \text { as } t_{2} \rightarrow t_{1} .
\end{aligned}
$$

Thus $\left\{(\mathcal{T} u): u \in B_{r}\right\}$ and $\left\{D_{0+}^{\gamma}(\mathcal{T} u): u \in B_{r}\right\}$ are equicontinuous and relatively compact in $C([0,1], R)$ by the Arzela-Ascoli theorem. Therefore $\mathcal{T}\left(B_{r}\right)$ is a relatively compact subset of $C_{\gamma}([0,1], R)$ and the operator $\mathcal{T}$ is compact.

Step 3: Let $u=\lambda(\mathcal{T} u)$ and $u=\lambda\left(D_{0+}^{\gamma}(\mathcal{T} u)\right)$ for $0<\lambda<1$. For each $t \in[0,1]$, define $\overline{\mathcal{M}}=$ $\left\{\|u\|_{\gamma} \in C_{\gamma}([0,1], R),\|u\|_{\gamma}<\mathcal{N}\right\}$. Then we get

$$
\begin{aligned}
\|u\|_{c} & =\|\lambda(\mathcal{T} u)\|_{c} \\
& \leq \varphi\left(\|u\|_{\gamma}\right) l_{\mathcal{T}_{0}} \Delta_{1}+\Delta_{g} \psi_{1}\left(\|u\|_{\gamma}\right)\left\|l_{g}\right\|_{1}+\Delta_{h_{1}} \psi_{2}\left(\|u\|_{\gamma}\right)\left\|l_{h}\right\|_{1}, \\
\|u\|_{c} & =\left\|\lambda\left(D_{0+}^{\gamma}(\mathcal{T} u)\right)\right\|_{c} \\
& \leq \varphi\left(\|u\|_{\gamma}\right) l_{\mathcal{T}_{0}} \Delta_{2}+\Delta_{h_{2}} \psi_{2}\left(\|u\|_{\gamma}\right)\left\|l_{h}\right\|_{1} .
\end{aligned}
$$

Thus

$$
\|u\|_{\gamma} \leq \varphi\left(\|u\|_{\gamma}\right) l_{\mathcal{T}_{0}} \sum_{i=1}^{2} \Delta_{i}+\psi_{1}\left(\|u\|_{\gamma}\right)\left\|l_{g}\right\|_{1} \Delta_{g}+\psi_{2}\left(\|u\|_{\gamma}\right)\left\|l_{h}\right\|_{1} \sum_{i=1}^{2} \Delta_{h_{i}} .
$$

That means

$$
\frac{\|u\|_{\gamma}}{\varphi\left(\|u\|_{\gamma}\right) l_{\mathcal{T}_{0}} \sum_{i=1}^{2} \Delta_{i}+\psi_{1}\left(\|u\|_{\gamma}\right)\left\|l_{g}\right\|_{1} \Delta_{g}+\psi_{2}\left(\|u\|_{\gamma}\right)\left\|l_{h}\right\|_{1} \sum_{i=1}^{2} \Delta_{h_{i}}} \leq 1 .
$$

For a non-negative $\mathcal{N}$ and $\|u\|_{\gamma}<\mathcal{N}$, the operator $\mathcal{T}$ which is defined in $\overline{\mathcal{M}}$ to be $C_{\gamma}([0,1], R)$ is continuous and compact. Therefore $\mathcal{T}$ has a fixed point in $\overline{\mathcal{M}}$.

\section{Examples}

Example 10 Consider the following boundary value problem of a fractional differential equation:

$$
\left\{\begin{array}{l}
D_{0+}^{\frac{3}{2}}\left(\phi_{p} D_{0+}^{\frac{3}{2}} u\right)(t)=l_{f}\left(\frac{|u(t)|}{|u(t)|+1}+\frac{\left|D_{0+}^{\frac{3}{2}} u(t)\right|}{\left|D_{0+}^{\frac{3}{2}} u(t)\right|+1}\right) \\
u(0)+0.1 u(1)=0.01 \int_{0}^{1} \frac{u(s)}{(1+s)^{2}} d s \\
u^{\prime}(0)+0.1 u^{\prime}(1)=0.01 \int_{0}^{1}\left(\frac{e^{s} u(s)}{1+2 e^{s}}+\frac{1}{2}\right) d s .
\end{array}\right.
$$

Here

$$
\begin{array}{ll}
\alpha, \beta=1.5, \quad \mu_{1}, \mu_{2}=0.1, \quad \sigma_{1}, \sigma_{2}=0.01, \\
v, \eta=0.3, \quad \tau=0.4, \quad \gamma=0.01,
\end{array}
$$

and

$$
f(t, u, v)=\frac{|u|}{|u|+1}+\frac{|v|}{|v|+1}
$$




$$
g(t, u)=\frac{u}{(1+s)^{2}}, \quad h(t, u)=\frac{e^{s} u}{\left(1+2 e^{s}\right)}+\frac{1}{2} .
$$

Since $0.88<\Gamma(1.5)<0.89, \Gamma(2)=1, \Gamma(2.5)=1$, we find

$$
\begin{aligned}
& \Delta_{1}=0.89, \quad \Delta_{2}=0.82, \quad \Delta_{g}=0.009, \\
& \Delta_{h_{1}}=0.0099, \quad \Delta_{h_{2}}=0.009, \quad l_{g}=l_{h}=1,
\end{aligned}
$$

with simple calculations. Therefore

$$
\begin{aligned}
& \left\{l_{\mathcal{T}_{0}}\left(\Delta_{1}+\Delta_{2}\right)+2 \Delta_{g}\left\|l_{g}\right\|_{1}+\left(\Delta_{h_{1}}+\Delta_{h_{2}}\right)\left\|l_{h}\right\|_{1}\right\} \\
& \quad<1.73 l_{\mathcal{T}_{0}}+0.04 \\
& \quad<1
\end{aligned}
$$

Then we can choose

$$
l_{\mathcal{T}_{0}}<0.562
$$

Thus all assumptions of Theorem 8 satisfied. Therefore the problem has a unique solution on $[0,1]$.

Example 11 Consider the following boundary value problem of fractional differential equation:

$$
\left\{\begin{array}{l}
D_{0+}^{\frac{3}{2}}\left(\phi_{p} D_{0+}^{\frac{3}{2}} u\right)(t)=\frac{|u(t)|^{3}}{9\left(|u(t)|^{3}+3\right)}+\frac{\left|\sin D_{0+}^{\frac{3}{2}} u(t)\right|}{9\left(\sin D_{0+}^{\frac{3}{2}} u(t)+1\right)}+\frac{1}{12} \\
u(0)+0.1 u(1)=0,01 \int_{0}^{1} \frac{u(s)}{3(1+s)^{2}} d s \\
u^{\prime}(0)+0.1 u^{\prime}(1)=0,01 \int_{0}^{1} \frac{e^{u} u(s)}{3\left(1+e^{s}\right)^{2}} d s \\
D_{0+}^{\frac{3}{2}} u(0)=0 \\
D_{0+}^{\frac{3}{2}} u(1)=0,3 D_{0+}^{\frac{3}{2}} u(0,3)
\end{array}\right.
$$

where $f$ is given by

$$
f(t, u, v)=\frac{|u|^{3}}{9\left(|u|^{3}+3\right)}+\frac{|\sin v|}{9(\sin v+1)}+\frac{1}{12} .
$$

We have

$$
|f(t, u, v)| \leq \frac{|u|^{3}}{9\left(|u|^{3}+3\right)}+\frac{|\sin v|}{9(\sin v+1)}+\frac{1}{12}, \quad u \in R .
$$

Here

$$
\begin{array}{ll}
\alpha, \beta=1.5, & \mu_{1}, \mu_{2}=0.1, \quad \sigma_{1}, \sigma_{2}=0.01, \\
v, \eta=0.3, & \tau=0.4, \quad \gamma=0.01, \\
\Delta_{1}=0.89, & \Delta_{2}=0.82, \quad \Delta_{g}=0.009,
\end{array}
$$




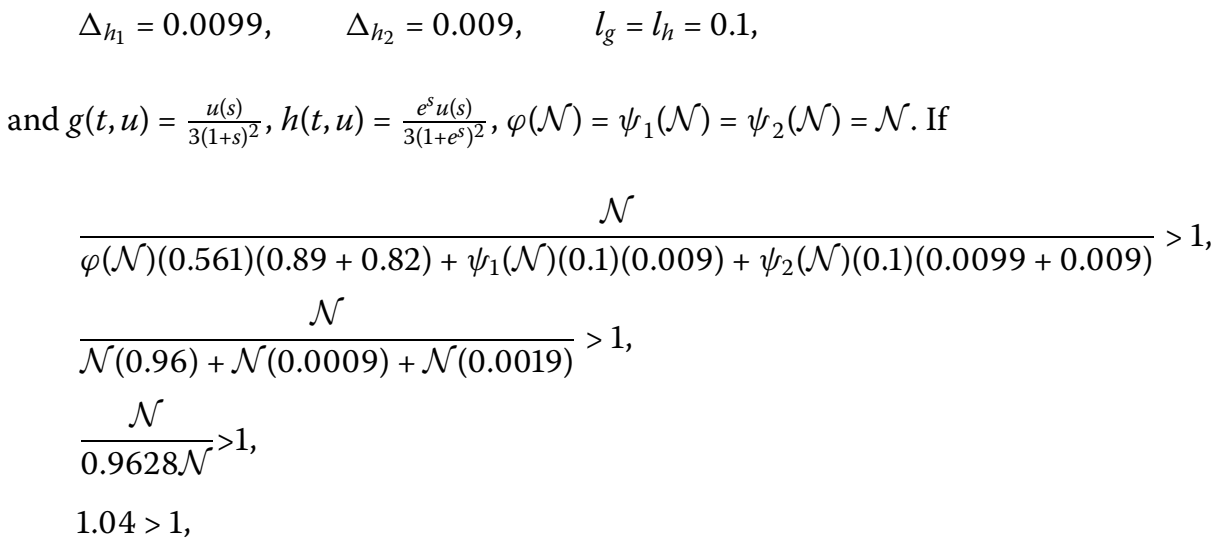

then (16) is satisfied. Then there exists at least one solution of the BVP on $[0,1]$.

\section{Competing interests}

The authors declare that they have no competing interests.

\section{Authors' contributions}

All authors contributed equally to the manuscript and typed, read, and approved the final manuscript.

\section{Acknowledgements}

The authors would like to thank the anonymous referees for their valuable comments and suggestions.

\section{Received: 19 March 2015 Accepted: 25 May 2015 Published online: 14 June 2015}

\section{References}

1. Wang, J, Xiang, H: Upper and lower solutions method for a class of singular fractional boundary value problems with p-Laplacian operator. Abstr. Appl. Anal. 2010, 971824 (2010)

2. Liu, X, Jia, M, Xiang, X: On the solvability of a fractional differential equation model involving the $p$-Laplacian operator. Comput. Math. Appl. 64, 3267-32777 (2012)

3. Ahmad, B: Nonlinear fractional differential equations with anti-periodic type fractional boundary conditions. Differ. Equ. Dyn. Syst. 21(4), 387-401 (2013)

4. Zhang, X, Liu, L, Wiwatanapataphee, B, Wu, Y: The eigenvalue for a class of singular $p$-Laplacian fractional differential equations involving the Riemann-Stieltjes integral boundary condition. Appl. Math. Comput. 235, 412-422 (2014)

5. Su, Y, Li, Q, Liu, X: Existence criteria for positive solutions of $p$-Laplacian fractional differential equations with derivative terms. Adv. Differ. Equ. 2013, 119 (2013)

6. Han, Z, Lu, H, Sun, S, Yang, D: Positive solutions to boundary-value problems of $p$-Laplacian fractional differential equations with a parameter in the boundary. Electron. J. Differ. Equ. 2012, 213 (2012)

7. Han, Z, Lu, H, Sun, S, Liu, J: Existence on positive solutions for boundary value problems of nonlinear fractional differential equations with p-Laplacian. Adv. Differ. Equ. 2013, 30 (2013). doi:10.1186/1687-1847-2013-30

8. Zhang, J, Liangand, S: Existence and uniqueness of positive solutions for integral boundary problems of nonlinear fractional differential equations with p-Laplacian operator. Rocky Mt. J. Math. 44(3), 953-974 (2014)

9. Khan, RA, Khan, A, Samad, A, Khan, H: On existence of solutions for fractional differential equation with $p$-Laplacian operator. J. Fract. Calc. Appl. 5(2), 28-37 (2014)

10. Wang, L, Zhou, Z, Zhou, H: Positive solutions for singular $p$-Laplacian fractional differential system with integral boundary conditions. Abstr. Appl. Anal. 2014, 984875 (2014)

11. Wei, L, Duan, L, Agarwal, RP: Existence and uniqueness of the solution to integro-differential equation involving the generelized p-Laplacian operator with mixed boundary conditions. J. Math. 33, 1009-1018 (2013)

12. Wei, L, Agarwal, RP, Wong, PJY: Study on integro-differential equation with generalized $p$-Laplacian operator. Bound. Value Probl. 2012, 131 (2012)

13. Wei, L, Agarwal, RP: Discussion on the existence of solutions to nonlinear boundary value problems with generalized p-Laplacian operator. Acta Math. Sci. Ser. A Chin. Ed. 32(1), 201-211 (2012)

14. Wei, L, Zhou, H, Agarwal, RP: Existence of solutions to nonlinear Neumann boundary value problems with p-Laplacian operator and iterative construction. Acta Math. Appl. Sinica (Engl. Ser.) 27, 463-470 (2011)

15. Wei, L, Agarwal, RP, Wong, PJY: Existence of solutions to nonlinear parabolic boundary value problems with generalized $p$-Laplacian operator. Adv. Math. Sci. Appl. 20(2)، 423-445 (2010)

16. Wei, L, Agarwal, RP: Existence of solutions to nonlinear Neumann boundary value problems with generalized p-Laplacian operator. Comput. Math. Appl. 56(2), 530-541 (2008)

17. Agarwal, RP, O'Regan, D, Papageorgiun, NS: On the existence of two nontrivial solutions of periodic problems with operators of $p$-Laplacian type. Differ. Equ. 43(2), 157-163 (2007)

18. Jiang, DQ, O'Regan, D, Agarwal, RP: A generalized upper and lower solution method for singular discrete boundary value problems for the one-dimensional $p$-Laplacian. J. Appl. Anal. 11(1), 35-47 (2005)

19. Aktuğlu, H, Özarslan, MA: Solvability of differential equations of order $2<\alpha \leq 3$ involving the $p$-Laplacian operator with boundary conditions. Adv. Differ. Equ. 2013, 358 (2013) 
20. Kong, X, Wang, D, Li, H: Existence of unique positive solution to a two-point boundary value problem of fractional-order switched system with $p$-Laplacian operator. J. Fract. Calc. Appl. 5(2), 9-16 (2014)

21. Khan, RA, Khan, A, Samad, A, Khan, H: On existence of the solution for fractional differential equation with $p$-Laplacian operator. J. Fract. Calc. Appl. 5(2), 28-37 (2014)

22. Hu, Z, Liu, W, Liu, J: Existence of solutions for a coupled system of fractional $p$-Laplacian equations at resonance. Adv. Differ. Equ. 2013, 312 (2013)

23. Chang, X, Qiao, Y: Existence of periodic solutions for a class of $p$-Laplacian operators. Bound. Value Probl. 2013, 96 (2013)

24. Chen, T, Liu, W: Solvability of some boundary value problems for fractional $p$-Laplacian equation. Abstr. Appl. Anal. 2013, 432509 (2013)

25. Chen, T, Liu, W, Liu, J: Existence of solutions for some boundary value problems of fractional $p$-Laplacian equation at resonance. Bull. Belg. Math. Soc. Simon Stevin 20, 503-517 (2013)

26. Liu, Y, Qian, T: Existence of solutions of periodic-type boundary value problems for multi-term fractional differential equations. Math. Methods Appl. Sci. (2012). doi:10.1002/mma.2746

27. Tang, X, Yang, C, Liu, Q: Existence of the solutions of two-point boundary value problems for fractional $p$-Laplacian differential equations at resonance. J. Appl. Math. Comput. 41, 119-131 (2013). doi:10.1007/s12190-012-0598-0

\section{Submit your manuscript to a SpringerOpen ${ }^{\circ}$ journal and benefit from:}

- Convenient online submission

- Rigorous peer review

Immediate publication on acceptance

- Open access: articles freely available online

- High visibility within the field

- Retaining the copyright to your article 